\title{
Ambrisentan use in a HIV-1 infected patient with end-stage renal disease and pulmonary hypertension: minimal removal by hemodialysis - a case report
}

\author{
José Ramón Santos ${ }^{1,2^{*}}$ (D) Ana Merino ${ }^{3,4}$, Walter E. Haefeli ${ }^{5}$, Cristina Miranda ${ }^{1}$, Marisol Prats ${ }^{6}$, loana Bancu ${ }^{3,4}$,
} Lucía Bailón ${ }^{1}$ and José Moltó ${ }^{1,2}$

\begin{abstract}
Background: Ambrisentan is a selective endothelin receptor antagonist used for the treatment of pulmonary arterial hypertension (PAH). Little is known about ambrisentan removal by hemodialysis in patients with end-stage renal disease (ESRD).

Case presentation: A 53-year-old woman with HIV/hepatitis $\mathrm{C}$ virus (HCV) co-infection, PAH and ESRD on regular hemodialyis was admitted in our hospital due to refractory heart failure while on treatment with bosentan (125 mg twice daily) and tadalafil (20 mg once daily) for PAH and antiretroviral treatment (CART) including darunavir/ cobicistat (800/150 mg once daily). Excessive exposure to bosentan due to drug interactions between bosentan and darunavir/cobicistat was suspected. Bosentan was replaced by ambrisentan, with progressive improvement in her clinical condition. Pre- and postdialyzer cocentrations of ambrisentan in plasma were determined and hemodialysis extraction ratio for ambrisentan was $2 \%$.
\end{abstract}

Conclusions: Our results suggest that hemodialysis results in minimal ambrisentan removal, and therefore no specific ambrisentan dosage adjustment seems to be required in ESRD patients undergoing hemodialysis.

Keywords: Ambrisentan, Hemodialysis, Pulmonary arterial hypertension, HIV-infection, Drug interactions

\section{Background}

The life expectancy of people living with HIV has shown significant improvement in recent decades, largely due to the widespread use of combined antiretroviral therapy (cART). However, this has been followed by an increase in the prevalence of non-AIDS-defining co-morbidities, including pulmonary arterial hypertension (PAH) and chronic kidney disease $[1,2]$. The prevalence of PAH in the HIV population is approximately $0.5 \%$, with a little variation since the introduction of cART. Nevertheless, the number of HIV-infected patients with end-stage renal disease (ESRD) requiring renal replacement therapy has increased.

\footnotetext{
* Correspondence: jrsantos@flsida.org

${ }^{1}$ Fundacio Lluita contra la SIDA, Barcelona, Spain

${ }^{2}$ Infectious Diseases Department, Hospital Universitari Germans Trias i Pujol,

Ctra de Canyet, s/n, 08916 Badalona, Barcelona, Spain

Full list of author information is available at the end of the article
}

Defining optimal dosing of many drugs in patients on hemodialysis may be difficult. Drug removal by hemodialysis is an essential factor in determining different dosing regimens. However, the pharmacokinetics of many drugs in patients on hemodialysis has been poorly studied, and information about their hemodialyzability remains scant [3-5]. In addition, HIV patients with $\mathrm{PAH}$ or ESRD are frequently affected by other co-morbidities that require specific therapies. These patients often receive many different drugs (polypharmacy), with a potential risk for drug-drug interactions among them [6, 7]. Here we report a clinical case involving an HIV-1infected patient with severe PAH and ESRD on hemodialysis who was treated with ambrisentan with the intention to avoid drug interactions between bosentan and cART. Ambrisentan is a selective endothelin-A receptor antagonist, which is approved for the treatment 
of PAH $[8,9]$. However, despite its better pharmacokinetic profile in comparison to bosentan, no data on ambrisentan pharmacokinetics in hemodialysis patients were available, so we evaluated the effect of hemodialysis on ambrisentan concentrations.

\section{Case presentation}

The patient in this clinical case was a 53-year-old woman who was admitted to our hospital with severe respiratory failure. She had been diagnosed with HIV/ hepatitis C virus (HCV) co-infection in 2000 as well as with PAH in 2002. In addition, she had ESRD and had been undergoing high-flux hemodialysis sessions since 2014 (vascular access: venous-arterial fistula; dialyzer: Revaclear ${ }^{\circ} 400$, capillary 101 dialyzer, membrane area $1.8 \mathrm{~m}^{2}$; Bicart ${ }^{\circ}$ Gambro bicarbonate cartridge; blood flow: $350 \mathrm{~mL} / \mathrm{min}$; dialysate flow: $500 \mathrm{~mL} / \mathrm{min}$ ). The patient was undergoing 3 dialysis sessions per week and each session lasted approximately $4 \mathrm{~h}$.

In January 2018, the patient was receiving cART with lopinavir/ritonavir (400/100 mg twice daily) plus raltegravir (400 mg twice daily), as well as bosentan (125 mg twice daily) plus tadalafil ( $20 \mathrm{mg}$ once daily) for PAH. At that time, treatment with glecaprevir/pibrentasvir was indicated for $\mathrm{HCV}$. To avoid drug interactions with HCV drugs, cART was switched to raltegravir $(400 \mathrm{mg}$ twice daily) plus rilpivirine ( $25 \mathrm{mg}$ once daily), and bosentan and tadalafil were temporarily discontinued. In April 2018, after completing 8 weeks of HCV treatment, bosentan and tadalafil were reintroduced at the same doses as before $\mathrm{HCV}$ treatment, and rilpivirine was replaced by darunavir/cobicistat (800/150 mg once daily).

From April 2018 to September 2018 the patient had several episodes of congestive heart failure, which were satisfactory managed with medical treatment. Nevertheless, in September 2018 the patient presented with severe respiratory failure and clinical signs compatible with decompensated heart failure, and she had to be hospitalized. On her admission, the patient underwent daily hemodialysis sessions, and the negative fluid balance in each session was increased. However, the response to hemodialysis was poorer than expected, suggesting additional determinants of her clinical situation. In addition, during the admission, virologic failure was evidenced by an HIV-1 RNA load in plasma of 5424 copies/mL, with HIV evolution and development of new drug resistance mutations in the integrase gene $(\mathrm{N} 155 \mathrm{H})$ not present in previous genotypes. After ruling out other conditions, bosentan toxicity-probably because excessive exposure to bosentan due to drug interactions between bosentan and darunavir/cobicistat was considered. Bosentan was therefore replaced by ambrisentan with the intention of avoiding drug-drug interactions with cART. Additionally, cART was optimized, and the regimen was changed to darunavir/ritonavir (800/100 $\mathrm{mg}$ once daily) plus etravirine (200 mg twice daily) and dolutegravir (50 mg twice daily). Thereafter, the patient experienced progressive improvement in her clinical condition, HIV RNA in plasma was re-suppressed $(<40$ copies $/ \mathrm{ml}$ ), and she was finally discharged to resume her usual hemodialysis program (3 sessions per week).

To evaluate the effect of hemodialysis on ambrisentan plasma concentrations, paired samples of blood entering ('in') and leaving ('out') the dialyzer and of the resulting dialysate were collected during one dialysis session. Blood samples were collected into heparin-lithiumcontaining 4-ml tubes $12.2 \mathrm{~h}$ after last ambrisentan dose. Plasma was isolated by centrifugation $(3200 \mathrm{~g}$ for 15 min), and stored at $-80^{\circ} \mathrm{C}$ until analysis. Dialysate $(20$ $\mathrm{ml}$ ) was directly collected from the dialysate port of the dialyzer. Ambrisentan concentrations in both plasma and dialysate were determined using high-performance liquid chromatography coupled with tandem mass spectrometry (LC-MS/MS) using a validated method [10], and the hemodialysis extraction ratio (ER) for ambrisentan was calculated following standard procedures [5]. The patient gave and signed an informed consent before sampling took place.

The effect of hemodialysis on ambrisentan concentrations was evaluated during one hemodialysis session. Pre- and postdialyzer total ambrisentan concentrations were $42.8 \mathrm{ng} / \mathrm{mL}$ and $42.5 \mathrm{ng} / \mathrm{mL}$, respectively, with negligible ambrisentan concentrations in dialysate $(3.92 \mathrm{ng} /$ $\mathrm{mL}$ ). The hemodialysis extraction ratio was only $2.0 \%$. At the end of the dialysis session (26.3 h after dosing), total ambrisentan concentrations in plasma were 35.6 $\mathrm{ng} / \mathrm{ml}$.

\section{Discussion and conclusions}

The small differences between plasma ambrisentan concentrations going in and coming out of the dialyzer, together with barely noticeable ambrisentan concentrations in dialysate in this patient, suggest a minimal removal of ambrisentan by hemodialysis in patients with ESRD. This finding is consistent with the physiochemical characteristics of ambrisentan, which is $99 \%$ bound to plasma proteins, and is minimally eliminated by the kidneys, with only $3 \%$ of its dose being excreted unchanged into urine [8].

Besides the effect of hemodialysis on ambrisentan concentrations, the clinical case presented here highlights the complexity of drug-drug interactions affecting HIVinfected patients. In this case, mutual interactions between bosentan and cART were suspected. The patient had clinical signs of fluid retention associated with bosentan toxicity, which was presumably the result of excessive bosentan concentrations due to potent cytochrome P450 3A4 (CYP3A4) inhibition by cobicistat. Although no specific studies have evaluated the effect of 
cobicistat on bosentan pharmacokinetics, data with lopinavir/ritonavir have shown a rather substantial $>5$-fold increase in bosentan exposure [11]. In the other direction, bosentan induces CYP3A4 activity [12], which is the main metabolic pathway for both darunavir and cobicistat. Previous studies have shown a decline in cobicistat exposure, and consequently inappropriately low darunavir concentrations, when darunavir/cobicistat is combined with the CYP3A4 inducers [13]. Consequently, combining bosentan with darunavir/cobicistat plus raltegravir could have resulted in functional monotherapy with raltegravir, leading to the virological failure and the development of new resistance mutations observed in this patient. Unfortunately, although it seems the most plausible hypothesis, no plasma samples to determine bosentan and antiretrovirals concentrations were available. Nevertheless, progressive improvement in the clinical condition of the patient along with re-suppression of HIV RNA after switching to ambrisentan and ART adjustment is concordant with our theory.

Ambrisentan is a selective endothelin-A receptor antagonist with proven efficacy and safety in the treatment of PAH [8]. In contrast to bosentan, ambrisentan is only a minor substrate of CYP3A4 and its elimination is mainly mediated by glucuronidation via several uridine-glucuronosyltransferase (UGT) isozymes [8]. Thus, the effect of potent CYP3A4 inhibitors on ambrisentan pharmacokinetics is much less compared to bosentan, and ambrisentan may be safely used in this scenario, as observed in our patient. Moreover, ambrisentan does not have any significant effect on the activity of UGT or cytochrome P450 isozymes or drug transporters, limiting the possibility of drug-drug interactions. It is noteworthy that ambrisentan concentrations at the end of the dialysis session were in the same range as those described in healthy volunteers receiving regular doses [14, 15], suggesting the absence of a need for dose adjustment in patients with ESRD.

Based on the minimal extraction ratio of ambrisentan by hemodialysis observed in our patient, no ambrisentan dose adjustment seems to be necessary in patients with PAH and ESRD undergoing hemodialysis. However, further investigations in this topic are recommendable. HIVinfected patients receiving polypharmacy may be affected by complex drug-drug interactions that require appropriate management, ideally by multidisciplinary teams with fluid communication among different prescribers.

\footnotetext{
Abbreviations

CART: Combined antiretroviral therapy; $C_{i n}$ : Predialyzer ambrisentan concentration; $C_{\text {out: }}$ Postdialyzer ambrisentan concentration; CYP3A4: Cytochrome P450 3A4; ER: Extraction ratio; ESRD: End-stage renal disease; HCV: Hepatitis C virus; LC-MS/MS: Liquid chromatography coupled with tandem mass spectrometry; PAH: Pulmonary arterial hypertension; TP: Total protein; UGT: Uridine-glucuronosyltransferase
}

\section{Acknowledgements}

We also wish to acknowledge the assistance of Michael Kennedy-Scanlon in proofreading the English in the final version of the manuscript.

\section{Authors' contributions}

J.R.S., J.M., I.B. conceived and contributed to the study design; J.R.S, A.M., I.B., M.P., L.B. contributed to the patient inclusion and clinical management; J.M., W.E.H. contributed to evaluate ambrisentan plasma concentrations and to calculate the extraction ratio; C.M., A.M. contributed to sampling and data collection; J.R.S, A. M, W.E.H., M.P., I.B., L.B., J.M. contributed to the drafting of the manuscript and provided critical revisions of the manuscript. The authors had full access to the data, and the corresponding author had the final responsibility for submitting the manuscript for publication.

\section{Funding}

This work was supported by the Fundació Lluita contra la SIDA. The funder had no role in study design, data collection and analysis, interpretation of data, decision to publish, or preparation of the manuscript.

\section{Availability of data and materials}

The datasets used and/or analysed during the current study are available from the corresponding author on reasonable request.

\section{Ethics approval and consent to participate}

Not Applicable.

\section{Consent for publication}

Written informed consent was obtained from the patient for publication of this Case Report and any accompanying images and videos. A copy of the written consent is available for review by the Editor of this journal.

\section{Competing of interests}

WEH reports having received personal fees, non-financial support, or other support such as research grants from Actelion $\mathrm{GmbH}$, Aspen Europe $\mathrm{GmbH}$, AstraZenica GmbH, BayerPharma AG, Berlin-Chemie AG, Boehringer GmbH, GSK, Bristol-Myers Squibb GmbH, Chiesi GmbH, Daiichi Sankyo GmbH, MSD Sharp \& Dohme GmbH, Roche, Grünenthal GmbH, Novartis, Pfizer GmbH, and personal fees and other from Roche GmbH, Smooth ClinicalTrials Ltd., Sumaya Biotec GmbH \& Co. KG, and Vaximm GmbH. JRS and JM have received research funding, consultancy fees, and lecture sponsorships from and have served on advisory boards for various laboratories (MSD, Abbvie, Boehringer Ingelheim, Gilead Sciences, Viiv Healthcare, Janssen Cilag, and Bristol-Myers-Squibb).

$A M, C M, M P, L B$, and IB have no conflicts of interest to declare.

\section{Author details}

${ }^{1}$ Fundacio Lluita contra la SIDA, Barcelona, Spain. ${ }^{2}$ Infectious Diseases Department, Hospital Universitari Germans Trias i Pujol, Ctra de Canyet, s/n, 08916 Badalona, Barcelona, Spain. ${ }^{3}$ Universitat Autònoma de Barcelona, Barcelona, Spain. ${ }^{4}$ Nephrology Department, Hospital Universitari Germans Trias i Pujol, Barcelona, Spain. ${ }^{5}$ Department of Clinical Pharmacology and Pharmacoepidemiology, Heidelberg University Hospital, Heidelberg, Germany. ${ }^{6}$ Pulmonology Department, Hospital Universitari Germans Trias i Pujol, Barcelona, Spain.

Received: 1 August 2019 Accepted: 5 December 2019

\section{References}

1. Ando M, Yanagisawa N. Epidemiology, clinical characteristics, and management of chronic kidney disease in human immunodeficiency virusinfected patients. World J Nephrol. 2015;4(3):388-95.

2. Nadkarni GN, Konstantinidis I, Wyatt CM. HIV and the aging kidney. Curr Opin HIV AIDS. 2014;9(4):340-5.

3. Urata M, Narita Y, Fukunaga $M$, et al. Simple formula for predicting drug removal rates during hemodialysis. Ther Apher Dial. 2018;22(5):485-93.

4. Hirata S, Izumi S, Furukubo T, et al. Factors influencing the removal rate of drugs by hemodialysis. J Jpn Soc Dial Ther. 2004;37:1893-900.

5. Molto J, Graterol F, Miranda C, et al. Removal of dolutegravir by hemodialysis in HIV-infected patients with end-stage renal disease. Antimicrob Agents Chemother. 2016;60(4):2564-6.

6. Ware D, Palella FJ Jr, Chew KW, et al. Prevalence and trends of polypharmacy among HIV-positive and negative men in the multicenter AIDS cohort study from 2004 to 2016. PLoS One. 2018;13(9):e0203890. 
7. Núñez-Núñez M, Castañeda-Macías I, Sandoval-Fernández Del Castillo S. Potential interactions in a cohort of elderly hiv-positive patients. Farm Hosp. 2018;42(4):163-7.

8. Ambrisentan (Letairis) prescribing information. Available at https://www. accessdata.fda.gov/drugsatfda_docs/label/2015/022081s033lbl.pdf.

9. Sise ME, Courtwright AM, Channick RN. Pulmonary hypertension in patients with chronic and end-stage kidney disease. Kidney Int. 2013;84:682-92.

10. Enderle $Y$, Witt $L$, Wilkens $H$, et al. Simultaneous quantification of endothelin receptors antagonists and phosphodiesterase 5 inhibitors currently used in pulmonary artery hypertension. J Pharm Biomed Anal. 2017;143:291-8.

11. Dingemanse J, van Giersbergen PL, Patat A, Nilsson PN. Mutual pharmacokinetic interactions between bosentan and lopinavir/ritonavir in healthy participants. Antivir Ther. 2010;15(2):157-63.

12. Bosentan Tracleer prescribing information. Available at https:/www accessdata.fda.gov/drugsatfda_docs/label/2017/209279s000lbl.pdf.

13. Moltó J, Curran A, Miranda C, et al. Pharmacokinetics of darunavir/cobicistat and etravirine alone and co-administered in HIV-infected patients. J Antimicrob Chemother. 2018;73(3):732-7.

14. Markert C, Hellwig R, Burhenne J, et al. Interaction of ambrisentan with clarithromycin and its modulation by polymorphic SLCO1B1. Eur J Clin Pharmacol. 2013;69(10):1785-93.

15. Markert C, Kastner IM, Hellwig R, et al. The effect of induction of CYP3A4 by St John's wort on ambrisentan plasma pharmacokinetics in volunteers of known CYP2C19 genotype. Basic Clin Pharmacol Toxicol. 2015;116(5):423-8.

\section{Publisher's Note}

Springer Nature remains neutral with regard to jurisdictional claims in published maps and institutional affiliations.

Ready to submit your research? Choose BMC and benefit from:

- fast, convenient online submission

- thorough peer review by experienced researchers in your field

- rapid publication on acceptance

- support for research data, including large and complex data types

- gold Open Access which fosters wider collaboration and increased citations

- maximum visibility for your research: over $100 \mathrm{M}$ website views per year

At $\mathrm{BMC}$, research is always in progress.

Learn more biomedcentral.com/submissions 